\title{
A Survey of EE Optimization Methods for Primary and Secondary Users in Cognitive Radio Networks
}

\author{
Tian Yang \\ Institute Charles Delaunay, University of Technology of Troyes \\ 12, Rue Marie Curie, 10300, Troyes (France) \\ E-mail: tian.yang@utt.fr \\ Moez Esseghir, Lyes Khoukhi \\ Institute Charles Delaunay, University of Technology of Troyes \\ 12, Rue Marie Curie, 10300, Troyes (France) \\ moez.esseghir@utt.fr, lyes.khoukhi@utt.fr
}

Received: July 21, $2018 \quad$ Accepted: October 13, $2018 \quad$ Published: October 31, 2018

DOI: 10.5296/npa.v10i3.13807

URL: https://doi.org/10.5296/npa.v10i3.13807

\begin{abstract}
A large scale of spectrum sensing techniques are proposed to improve the use of spectrum resources. However, the EE (energy efficiency) should be guaranteed for both primary and secondary users, especially under various detection performance constraints. In this regard, the linking between activities of PU (primary user) and dynamic access behaviors of SUs (secondary users) should be considered in an integrated way. This survey has compared different existing scenarios and frameworks on EE optimizations. The principal objective is to enhance the system throughput and to coordinate on the physical layer of both PU and SUs, in order to enable a high-quality spectrum detection and a more efficient spectrum access. In the technical part, several optimization methods are introduced under PU's constraints, and different methods based on game theory are applied to suitable cooperative sensing scenarios for SUs' optimal access. Finally, the complexity of algorithms is compared, to further reduce the execution time and deploy real-time adaptation for users with lower delay.
\end{abstract}

Keywords: Spectrum sensing; Cognitive radio; Energy efficiency; Optimization methods; Game theory; Dynamic spectrum access. 


\section{Introduction}

A large number of technique schemes were proposed on accurate and efficient spectrum sensing in CRN (Cognitive Radio Networks). These contributions can be roughly divided into three categories:

1) Various sensing techniques and scheduling schemes are proposed to improve the SE (Spectrum Efficiency) by properly reusing the spectrum resources of primary users; besides, different EE (Energy Efficiency) functions are formulated as the targets of optimization problems (i.e., utility functions) to enhance the system performance under certain constraints.

2) PU activities towards the reawake probability, detection constraints, collisions, interferences, QoS provisioning, etc. These aspects can act as the conditions of an optimization formulation. On the other side of sensing, the main focus is on SU's frame structure including optimized sensing time ratio, sensing threshold and fusion rules.

3) SU's access actions and transmission. For example, when and how should a SU access to the channel and which channel to access in order to achieve the optimal transmission. SU's access strategies are usually discussed in a cooperative spectrum sensing (CSS) model, in which each SU act as different roles according to their shares of the aggregative sensing contribution.

In this paper, various spectrum sensing schemes are explained with examples. Several typical works on the basis of DSA (Dynamic Spectrum Access) concept have been presented. These works provide a large scale of techniques and frameworks of CRNs, and they especially propose solutions towards the efficient spectrum usage. Moreover, we analyze different methodologies against different scenarios of EE optimizations. The main focuses of EE formulations are compared in detail, especially under different constraints (e.g. interference, collision, PU protection, etc.). Afterwards, our comments and propositions have also been added towards the drawbacks of these works. Finally, the complexities and performances of related algorithms of these methods are evaluated and compared.

The reminder of our survey is arranged as follows: Section 2 presents novel techniques and scenarios that reflect the core target of CRN, which is the accurate and efficient reuse of spectrum resources. It also explicates that PU and SU targets are correlated and interactive, several variables/constraints should be jointly optimized from the both sides. Taking into account the impact of PU's activities, Section 3 mainly contributes to the formulation of EE optimization problems, the classification of PU's performance targets, and the comparison of complexities of typical PU-oriented algorithms. Section $\mathbf{4}$ focuses on the operations and reactions from SUs' side. This section notably refers to the power allocation problem, and the division of sensing/transmission time ratio, etc. Different game theory methods are illustrated in this section in order to optimize the related performance metrics of SUs. Section 5 presents the prospects of recent works which integrate cross-layer methods and routing strategies into CRNs. Finally, Section 6 concludes the methods and techniques mentioned in our survey and introduces some future research directions as an overlook. 


\section{Sensing techniques and scenarios}

Many recent literatures focused on recent innovations and techniques of spectrum sensing. In this section, we introduce the principles of some typical related works and their scenarios.

In [1], the authors aim to optimize the dynamic spectrum access while guaranteeing sufficient PU protection. The paper proposes a new sensing scheme in which the sensing process is divided into two individual processes. A second sensing is performed to confirm the presence of PU in addition to the first sensing result, and to improve the sensing accuracy of the system. However, the additional expenditure of the second sensing is rather important to consider and the difficulty of synchronicity issue should also be noticed.

In [2], A. Azarfar et al. propose a mixed scheduling scheme of cooperative spectrum sensing to enhance the system throughput performance. For the sequential sensing technique, SUs take turns to sense the related channel, but the transmission time is determined by the access order. For this reason, the transmission duration appears to be random. For the parallel sensing technique, SUs can sense the channels simultaneously. However, the channels are not supposed to be accessed if no SUs contribute to the sensing, since the status of channels is unknown. In this regard, the authors employ a hybrid design of dynamic spectrum access schemes to optimize the opportunistic access chance and the average transmission duration, under detection constraints. Then, the performances of heuristic methods in three DSA techniques (i.e., parallel, sequential and hybrid) are analyzed and compared, the authors present the suitable occasions of these techniques and the sensing time was determined adaptively. As an advanced version of [2], A. Azarfar et al. [3] point out that the user assignment to channels should be considered in order to optimize the transmission performances. The paper analyzes the spectrum opportunities of each channel (i.e., channel vacancy) after the SUs take turns to sense scheduled by a DSA strategy. The paper also proposes heuristic algorithms to solve the tradeoff problem between cooperative sensing time occupation and remaining transmission duration, especially under certain user assignment and sensing schedule strategies. In addition, the received SNRs (Signal to Noise Ratio) are also considered and are associated to SUs while assigning channels to SUs, then SUs are scheduled to always pick the best SNR in a heterogeneous multi-channel CR network.

However, the proposed methods are only suitable to small-scale CRN. Even though the authors have considered the comparisons of computational complexity and memory storage, the CR networks with large number of channels and SUs could take rather long computational time to solve the optimization problem. Other work such like [4] proposes admission control DSA schemes based on states predictions in a discrete-time Markov chain model. The paper analyzes the cases of states transitions in order to launch channel handoff actions at suitable occasions, and SU's QoS is especially guaranteed by measuring the forced termination probability and minimizing this probability. However, the article focuses too much on the transition of states, without considering the major sensing factors such as the sensing threshold and the sensing time ratio.

The authors of [5] focus on the channel assignment and scheduling problem in a 
heterogeneous model. The authors investigate the optimization problem of PU-SU channel mapping. They notably consider the overall energy cost on switching, sensing, and reporting phases. Afterwards, by scheduling PU's available channels and controlling SU's channel search duration, they analyze the channel assignment matrix to optimize energy and spectrum efficiency targets under detection requirements. This work, however, combines too many targets on PU channels' scheduling problem (i.e, channel access, channel switch, channel search, etc.). Indeed, the PU's channel accesses have their randomness and this is due to probability distributions, therefore it is rather difficult to control the channel search duration in practice, not to mention to predict the latest arrival of SU. More attention should be paid to channels' states changes instead of only considering a static binary hypothesis for PU channels. [6] introduces PU's interference constraints into SUs' DSA design, the paper notably optimizes the transmission power under interferences and SINR requirements with two steps: (1) Firstly, the authors propose a differential evolution-based cooperative-game method on power allocation problem. (2) Secondly, they analyze the impact of sensing-phase length to further integrate power allocation algorithm with the DSA scheme for opportunistic access of SUs.

These aforementioned techniques mainly study the DSA strategies of users' channel access and assignment in multi-channel conditions, especially to make the most of spectrum opportunities and to optimize the efficiency of spectrum utilization. Nevertheless, since PU's presence is uncertain and unpredictable in practical channels, SUs could only obtain information from their detected results. In this regard, apart from the proposed scheduling methods, the distribution of PU's activities and corresponding performance metrics (e.g. SINR, error probabilities, etc.) should also be analyzed in detail.

A recent contribution [7] proposes a reconfiguration scheme of SINR predictions according to the dynamical channel gain. Then, the sampling size is notably adapted according to the feedback of channel state estimations. Authors of the paper also formulate sensing-throughput tradeoff and proposed schematic algorithm to optimize the sensing duration. Unlike other papers who only focus on scheduling the channel assignment, the advantage of [7] is that they consider PU's activities as a two-states Markov chain, and they especially study the interactive behavior between PU and SUs, which contributes to a better sensing quality.

Other papers concentrate on the integration of novel spectrum sensing techniques into EE maximization problem. The authors of [8] study CoMAC-based spectrum sensing scheme and propose to solve the EE maximization problem by jointly optimizing the sensing time and symbol sequence length. The sensing threshold is especially analyzed so as to define the boundary conditions of sensing performances. In [9], the authors adopt the continuous sensing by considering the total spectrum band of the sensing and the transmission period as a fixed number and allocated proper bandwidths and power, for each of two periods.

\section{Related works of PU-oriented EE optimizations}

Existing surveys as [10] have studied the modelling of PU activities based on Markov process and queuing theory. In contrast, our survey integrates the PU's reactivation into the 
formulation of system EE, and analyzes the optimization of power allocation by considering PU's activity under certain QoS requirements, based on proposed game theory and optimization methods (e.g., heuristic, bi-section search, etc.).

In this section, we introduce the related papers about the problematics of energy efficiency, especially the contributions which adapt primary variables (e.g. transmission power, PU presence, and performance achievement, etc.) on PU's side. In which follows, some tables are presented to indicate the focusing points of existing works, others are given to compare the performances and the complexities of related algorithms.

\subsection{Definitions of energy efficiency (EE) optimization}

Several works contribute to the formulation of EE problem. [11]-[16] and investigate the sensing-EE tradeoff problem. The sensing and transmission durations are adapted to the optimum while guaranteeing PU's detection at the same time. These papers aim to reduce the risks of collision and to prevent from SUs' greedy accesses. Similarly, the authors of [17] formulate a novel utility function by removing the miss detection loss and power consumption parts from throughput gain, then they optimize the utility in the proposed cooperative sensing framework. The authors of [18]-[20] propose to distribute proper sensing time to achieve the peak of EE function, some of the authors such as [18] applies the original form of EE defined as throughput per unit energy consumption. Then, they take into account the number of sensing performers, they apply suitable fusion rules and related energy detector thresholds to further improve the performances. [21] formulates the energy saving and the transmission time maximization as a bi-objective optimization, and it applies outer linearization methods to find optimal solutions. However, the paper only focuses on the scheduling problem on SU-channel pairs, whereas PU's presence activities have not been particularly considered. The detailed target-EE definitions and examples are listed in Table 1.

Table 1. Definitions of EE and related contributions in literature

\begin{tabular}{|c|c|}
\hline Reference & Main focus and contribution \\
\hline [1] & $\begin{array}{l}\text { It formulates EE as averaged throughput per energy during a frame time, where the analysis of a frame of SU is divided } \\
\text { into several cases of access actions determined by first and second sensing results. }\end{array}$ \\
\hline [15] & $\begin{array}{c}\text { It considers PU's reoccupation and SUs' interferences on PU when designing EE with the sensing and transmission } \\
\text { durations. }\end{array}$ \\
\hline [19] & $\begin{array}{l}\text { It studies the influences of network scale and fusion rule in EE maximization to find suitable and efficient CSS scenario for } \\
\text { SUs. }\end{array}$ \\
\hline [22] & $\begin{array}{l}\text { It selects a suboptimal transmission power of SU, which is a unimodal to averaged EE. Then, for the second optimization } \\
\qquad \text { of averaged EE, sensing time and detection threshold are jointly optimized. }\end{array}$ \\
\hline [23] & $\begin{array}{l}\text { It formulates the target function as a linear one with both unimodal properties of SE and EE being considered. The solution } \\
\qquad \text { of SE/EE tradeoff was obtained by optimizing both sensing time and threshold. }\end{array}$ \\
\hline [24] & $\begin{array}{l}\text { It focuses on EE formulation under transmit/interference power and collision constraints. Then, the paper is aimed to } \\
\text { maximizing the throughput under minimum EE requirements. It proposes power control schemes and adapts frame } \\
\text { duration to achieve this purpose. }\end{array}$ \\
\hline [25] & It proposes to solve the joint optimization of EE by adapting both sensing/transmission durations and transmission power. \\
\hline
\end{tabular}




\subsection{Primary user activities and the impacts on $E E$}

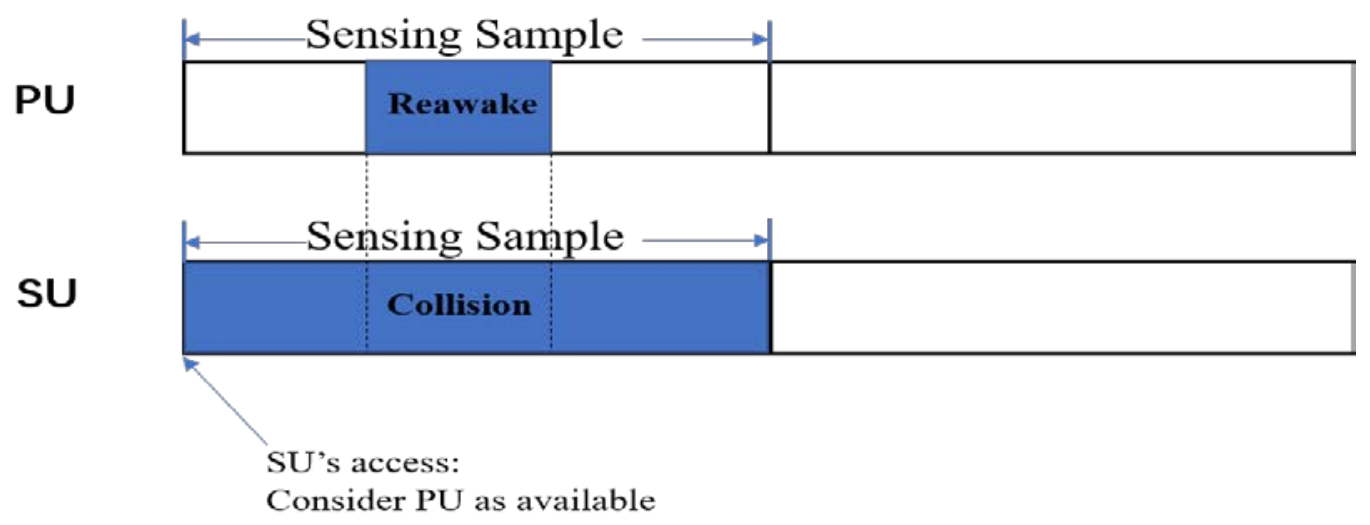

Figure 1. PU’s reawake probability considered in [3] and [15]

Some papers mainly discuss the influence of the transmission power on EE optimization with primary users. The authors of [3] prove that EE is a concave function to the transmission power, that an optimized power could be always found by iterative bi-section search. [22], [26] apply golden section methods to select an optimized transmission power, and to fix a proper sensing duration, respectively. The authors consider the EE together with optimized fusion rules. In addition, the authors of [15] and [27] consider the PU activities. Particularly, they study the reawake probability of PU during the transmission period. As PU's presence is subject to random process, the states' change of PU could increase the potential collision risks caused by miss detection (i.e., the collision due to PU's reawake is presented in figure 1), and eventually the system throughput could be decreased. Therefore, the reawake problem should be particularly noticed in practice. [28], [29] studied how to control PU's rate loss and its interferences imposed on SU's transmission, based on which power allocation schemes are designed to achieve higher transmission rate, while guaranteeing the sensing constraints being achieved. Other works such as [30] associated the power optimization problem with new admission price, where the rate constraints of both PUs and SUs are satisfied at the same time by applying a feasible access decision. However, associating new SUs to the current user set based on SUs' own spectrum radius is still debatable, since smaller radius also brings higher interference to current users. The spectrum radius seems to be less relevant to the core optimization problem (i.e., network capacity, power allocation, etc.); Otherwise more details should be given to better demonstrate that SU with shorter radius matches better with selected user set. [31] concentrates on selecting qualified SUs to perform the simultaneous spectrum co-access with PU by applying round robin access technique, while the rest of SUs can have opportunistic spectrum access through fair competitions if PU is in an idle state.

\subsection{Performance metrics, PU protection and SINR targets}

PU's protection and SINR provisioning are also widely discussed in related papers. Hereby, PU's protection stands for the performance targets such like detection probability, 
this is different from PU's aforementioned activities. In this section, PU's activities are normally considered as a constant, and we particularly analyze the impact of PU's detection constraints to the system performance (e.g., throughput). In Table 2, the principal aspects considered in two main categories (i.e., PU's activities and to PU's protections) are concluded together, to present and to classify the existing works and methods towards PU's design.

Table 2. Related researches on the primary users’ side

\begin{tabular}{|c|c|c|}
\hline \multirow{4}{*}{ PU's activities } & Technical Points & Reference Examples \\
\hline \hline \multirow{4}{*}{ PU's protections } & Reawake & [3], [15], [27], [32]-[33] \\
\cline { 2 - 3 } & [3], [22], [26], [34] \\
\cline { 2 - 3 } & Transmission Power & [28], [29], [34] \\
\hline & PU interference & [35]-[39] \\
\cline { 2 - 3 } & Detection Constraint & [32], [40]-[41] \\
\cline { 2 - 3 } & Collision Avoidance & [42]-[44] \\
\cline { 2 - 3 } & Traffic Load & [18], [45]-[47] \\
\hline
\end{tabular}

[10], [35]-[37], [48] mainly apply a lower bound of detection probability to guarantee the accuracy of detection, and consider other targets (e.g. EE, throughput, PU's spectrum utilization, etc.) under performance constraints. [18] presents the opportunistic access techniques to realize PU protection while maintaining SU's QoS at the same time. [42] regulates the queue length in order to control PU traffic load and thus satisfy the QoS provisioning of PU. [40] proposes a MAC-level protocol where SUs employ CSMA to determine the back-off time and to avoid collisions by monitoring the channel. Then, FSS (fast spectrum sensing) technique is particularly applied after the channel contention phase to confirm the detection of PU before the transmission. This scheme can improve the protection of PU at the cost of high expenditure of additional headers. The authors of [41] have evaluated the reward of each action and selected an optimized operation mode for each operating or backup channels, in order to avoid the disturbance of PU-existence channel to the data transmission. [49] utilizes spectrum sharing technique to compensate parts of SUs' power to PU and assist its transmission. Other papers (e.g., [8], [39], [50]-[51]) study the dynamic optimization of energy detector threshold to guarantee proper decisions of PU's signal and to reduce the total sensing error rate. Among them, a recent paper [51] has investigated the distribution of error probabilities related to PU's dynamic presence under certain sampling frequency, and then it formulates the problem as a two-state Markov chain with PU states changes.

\subsection{Algorithm analysis and complexity comparison}

In the context, a large number of papers have proposed diverse searching method to locate the optimum from different views of EE optimization. In this section, we mainly introduce golden section search [22], [26], bi-section search [3], [15], linear search methods 
[52], and heuristic method [21], [53]-[55]. The occasions and conditions of using these related methods are analyzed and the examples of application scenarios are given to each method. Then, with the introduction of the similar scenarios on EE optimizations, the performances of calculations are compared among these scenarios, and the algorithms' complexities are studied for each method.

Firstly, we introduce the water-filling algorithms which are suitable for resource management problems, especially in multi-subcarrier scenario. Water-filling is a set of methods who contribute to calculate the restored power level for each channel, and to allocate power resources accordingly based on the calculations of Lagrange patterns. For example, authors of [28], [54] and [56] consider the optimized power allocation scenarios in multiple subcarriers OFDM-based CR system. [56] applies water-filling form into bi-section search based on interference, power constraints and rate requirements. The authors first propose an original form of bi-section search to optimize power allocation of subcarriers using two searching loops. Then, by proving that Lagrange multiplier is a decreasing function of total transmission power P (i.e., lemma.5 of [56]), the original WFAS (water-filling factor aided search) algorithm is simplified by removing the outer search of $\mathrm{P}$. The simplification saves significant complexity of $\log 2\left(\mathrm{P}_{\mathrm{T}} / \varepsilon\right)$, where $\mathrm{P}_{\mathrm{T}}$ is the total maximum transmission power and $\varepsilon$ represents the accuracy of convergence. Similarly, [28] utilizes water filling based bi-section search to dynamically find the optimums of hybrid PU protections under rate loss constraints, and especially compares this method to conventional formulation with interference power constraints to highlight the advantage. However, the complexity is not improved for the proposed algorithm, the paper only discusses the breakthroughs on performance comparison.

In addition, other algorithms such as linear search and heuristic search also propose complementary strategies of EE optimized resource allocation problem in multi-channel system. Followed by a discretion step of transmit power and sensing duration, [52] develops a linear search method to find feasible solutions of EE function, subject to the sensing duration constraints. As the search list indicates, the complexity of linear search method equals to the product of length of both two axis (i.e., power and sensing time). Since linear search is based on traversing the entire search list, it is considered still more complex than binary search or hash tables which refer to sequential and fast research. To further shorten the computational time, authors of [52] also propose iterative-based algorithm to alternatively optimize the two targets. However, the solution of iterative based algorithm might be only a local optimum, and thus cannot be guaranteed as global optimum. The principal advantage of this work is supposed to be the reduction of computational time comparing to conventional exhaustive search.

Considering the channel assignment and channel switching problems, heuristic methods are proposed by several contributions to properly schedule on the diversity of CR users, in order to achieve the general maximization of EE. For instance, [21] focuses on the greedy channel assignment to SUs and especially proposes to save the sensing and reporting energy without breaking the sensing tasks. More specifically, the SUs with optimized SINR values are selected to make a match with a specific channel, and the remaining sensing time is 
updated for the next round. The loop continues until the minimum number of SUs contributing to cooperative sensing tasks is reached. However, the complexities of two proposed algorithms are still as high as $O(M N \log N)$, depending on the number of channels $\mathrm{M}$ and the number of SUs which is denoted by $N$. As a comparison, with a uniform and direct formulation of EE, [53] tentatively fulfills the transmission requests of SUs in their buffers upon frequency assignments, and the complexity is denoted as $O(F N)$, with $F$ being the number of CR channels. As an extensive work of [53], the authors of [55] apply heuristic algorithms to solve frequency-SU pairs matching problem in polynomial-time, and they propose more options of target functions such as throughput optimization under EE restrictions, or energy minimization subject to minimum throughput requirement, which are both considered as LP (linear integer programming) problem as a replacement to the original non-LP formulation of EE. The heuristic scheduling algorithm EEHS (energy-efficient heuristic scheduler) of [55] holds the same complexity as the one presented in [53].

Secondly, for a simplified channel scenario in CRNs which contains only one PU and SUs (i.e., without differentiating the resource allocations on each channel), the bi-section and the golden section searches are widely used. The objective of these searching methods is to locate the optimum under certain targets (e.g., transmission, energy efficiency, spectrum usage, interference, etc.) and within a relatively short computational time.

The first step to execute these unique-axis searching methods is to confirm the precondition of optimization, that is, to prove the target function is a concave one; or in the opposite cases, to convert the target problem into a concave function. Then, the searching range is obtained based on the analyzed restrictions on the other sub-targets. The golden section method [57] proposes to utilize the ratio of golden section to realize dynamic, proportional and fast search recursion. The recursive algorithm is designed based on the comparison of two golden section bounds calculated by the golden ratio distribution. The bound corresponding to smaller value on the target function will be defined as new bound of next loop (e.g., lower or upper bound). Then, the research is repeated until a convergence is found (i.e., the distance between upper and lower bound is controlled below a certain constant).

The bi-section and golden section methods are both based on iterative replacement of searching range, so the principles of these algorithms are similar. The complexities of algorithms are depended on the margin of searching range and related requirement of searching accuracy. These searching methods are especially useful while the initial function is well-known or at least the convexity is derivable. Besides, the target function should also hold the continuity so that a global optimum can be guaranteed to be existent. 
Table 3. Comparison of algorithm complexities in related works

\begin{tabular}{|c|c|c|c|}
\hline Methods & References & Complexities & Remarks \\
\hline \multirow{2}{*}{ Heuristic } & {$[21]$} & $O(M N \log N)$ & $\begin{array}{l}M \text { : number of channels, } \\
\quad N \text { : number of SUs }\end{array}$ \\
\hline & [53], [55] & $O(F N)$ & $\begin{array}{c}F \text { : number of CR channels } \\
\qquad N \text { : number of SUs }\end{array}$ \\
\hline \multirow{2}{*}{ Bi-section search } & [34] & $O\left(\log _{2} \frac{P_{t h}}{\epsilon_{p}} \log _{2} \frac{u_{\max }}{\epsilon_{u}}\right)$ & $\begin{array}{l}P_{t h}: \text { power threshold } \\
u_{\text {max }}: \text { maximum rate }\end{array}$ \\
\hline & [3] & $O\left(\log _{2} X \log _{2} \frac{\theta_{\max }-\theta_{\min }}{\epsilon_{\theta}}\right)$ & \multirow{3}{*}{$\begin{array}{c}{\left[\theta_{\min }, \theta_{\max }\right]:} \\
\text { range of sensing ratio. } \\
{\left[\phi_{\min }, \phi_{\max }\right]:} \\
\text { range of SU's power } \\
\epsilon_{\theta}, \epsilon_{p}: \text { unities of search } \\
X, Y \text { : resolution of search }\end{array}$} \\
\hline Linear Search & [52] & $O\left(X \log _{2} \frac{\phi_{\max }-\phi_{\min }}{\epsilon_{p}}\right)+O\left(Y \log _{2} \frac{\theta_{\max }-\theta_{\min }}{\epsilon_{\theta}}\right)$ & \\
\hline Exhaustive Search & [58], [59] & $O(X Y)$ & \\
\hline
\end{tabular}

To better compare the efficiency of algorithms proposed in each category, we present the complexities and generalize them in Table 3. It can be observed from this table that the complexities of searching methods are mostly depended on the variables and applied method. The references of both nested iteration methods and double-variables optimizations are introduced and compared in this table.

\section{Related works of SU-oriented EE optimizations}

Efficient spectrum utilization has become a great challenge to wireless networks as the diversity and service requirements of wireless services are both growing rapidly. Cooperative spectrum sensing (CSS) technique allows allocating available spectrum resources of a primary user to a group of secondary users (under certain rules) during its "spectrum hole" in order to increase the spectrum efficiency of the network [60], [61]. SUs have to conduct periodical sensing in order to monitor PU's current state, then a fusion center (FC) collects SUs' sensing results and makes access decisions for them accordingly.

Essential elements of CSS are introduced in [62], and the infrastructures of cooperative sensing are classified. However, the paper hasn’t compared among existing works and related methods. [63] presents detailed classifications for various system models of CSS, compares the EE formulations and performance targets in different scenarios, analyzes the advantages and drawbacks of some related techniques. In this section, we introduce and analyze the recent literatures about SUs' access strategies in CSS, and notably presents a classification of game theory methods [64] to complement the previous surveys. 


\subsection{Related works on cooperative spectrum sensing}

Table 4. Basic targets and main research scopes in CSS

\begin{tabular}{|c|c|}
\hline Technical Points & Reference Examples \\
\hline \hline Sensing Scheduling & {$[8],[61],[65]$} \\
\hline EE / Throughput & {$[66]-[68]$} \\
\hline Faireness & {$[65]$} \\
\hline Channel Assignments & {$[2],[21],[60]$} \\
\hline Error rate & {$[69],[70]$} \\
\hline
\end{tabular}

As presented in Table 4, existing contributions mainly focus on the expected throughput under a given fusion rule, or the sensing and accessing time distribution depended on the scheduling scheme (e.g., [60]). These contributions incent SUs to become contributors by setting reward functions, either to attract high-SNR SUs by allocating different sensing priorities [68], or to allocate more accessing possibilities to the channels in better condition [61], [71]. Other contributions consider throughput with miss detection probability in order to avoid collision between PUs and SUs and to guarantee the sensing performance. Fairness is guaranteed in [65] by allocating high priority to SUs which contributes to CSS, and by detecting selfish and malicious SUs which deviate from the equilibrium of general utility function. As a result, the SUs who have detected more accessing opportunities on their channels should contribute more to the cooperative sensing process. The authors in [69] investigate the total error rate in different channels and observe the optimized number of SUs for reaching an adequate sensing performance.

The authors in [61] propose a novel game to distribute SUs to choose from parallel sensing or cooperative sequential sensing, and to calculate the payoff accordingly. However, this method is not feasible in practice because the evolutionary game only allows determining the probability of contribution for SUs, and it is difficult to be used on making flexible choices for SUs between two existing techniques. In [66], the authors analyze the SUs' evolutionary stable strategy (ESS) in synchronous /asynchronous scenario where PU shares the same clock with SU (or not). Wang et al. [67] propose to optimize the throughput by dynamically selecting SU' status between "contributor" and "free-rider" based on the analysis of transmission gain. The authors of [68], [66], [67] focus on the optimization of energy and throughput, but they haven't considered impact of different detection performance. [72] focuses on maximizing the expected secrecy transmission rate over a period of time, by dynamically determining the actions of SUs. The opportunistic access allows SUs to save the energy of battery with the harvested energy technique. [73] applies coloring scheme to group SUs in two-tiers heterogeneous CR network. According to the distance between base stations, the authors determine the average number of CBS channels to be allocated from PU in order to achieve the QoS targets and to minimize the outage probability function. 
As a conclusion, the energy has been presented as the total energy consumption in most cases, which is related to the number of contributors. Most papers target other objectives (i.e. throughput, detection performance, channel selection, etc.) to control the number of contributors, then they compute average energy based on this number. However, spectrum waste of CSS should be considered with SU's actual sensing effort (i.e. the sensing time ratio [74]). Therefore, the energy should always be compensated for the spectrum waste to guarantee the performance.

\subsection{Typical SU-oriented methods and Game theory applications}

Firstly, we briefly classify the core existing techniques of SU reactions in aforementioned DSA schemes and in the cooperative sensing scenario. These strategies lead SUs to act as a proper role while having an opportunity of accessing to the spectrum. In DSA, SUs have to rapidly access/concede to the spectrum once receiving decision from a controller: either to be active to improve SE, or to stay quiet to avoid causing collisions with PU; In CSS, all of SUs behavior in a uniform way: Each user records the behaviors of other users, and choose to act as a "contributor" or a "free-rider" during the sensing process. The cooperative desires of SUs are dependent on the probability distribution and have randomness at the beginning, but they tend to have a stable behavior after the equilibrium of the game is achieved. Then, the accesses of SUs are based on the presence of PU and the quality of detections, according to the role that a SU is playing. The scenarios of SU's accessing criteria are presented for both DSA and CSS, and we notably analyze the characteristics of the core related techniques in Table 5 with some typical references as examples.

Table 5. Techniques and methods of SUs' operations in DSA and in CSS

\begin{tabular}{|c|c|c|c|c|}
\hline Methods & References & Advantages & Drawbacks & Application Areas \\
\hline SU association & $\begin{array}{c}\text { [30], [73], } \\
\quad[75]\end{array}$ & $\begin{array}{l}\text { High capacity } \\
\text { usage, and high } \\
\text { QoS performance }\end{array}$ & $\begin{array}{l}\text { Subject to mobility,reliability } \\
\text { and detection accuracy }\end{array}$ & $\begin{array}{l}\text { Dynamic spectrum } \\
\text { access (Hetnets) }\end{array}$ \\
\hline Clustering & $\begin{array}{c}{[60],} \\
{[76]-[77]}\end{array}$ & $\begin{array}{l}\text { Flexible, and low } \\
\text { complexity }\end{array}$ & $\begin{array}{l}\text { High scheduling and } \\
\text { operational costs }\end{array}$ & $\begin{array}{c}\text { Self-organization } \\
\text { scenarios, suitable for } \\
\text { heuristic algorithms }\end{array}$ \\
\hline \multirow{2}{*}{ Behavior dynamics } & \multirow{2}{*}{ [67], [78] } & \multirow{2}{*}{$\begin{array}{l}\text { Fast learning } \\
\text { solution with } \\
\text { reliable } \\
\text { performance }\end{array}$} & $\begin{array}{c}\text { Not applicable to more } \\
\text { complicated scenarios with } \\
\text { multi-constraints }\end{array}$ & \multirow{2}{*}{$\begin{array}{c}\text { A steady probabilistic } \\
\text { scenario for SUs to } \\
\text { fairly compete with } \\
\text { each other for uniform } \\
\text { spectrum usage in CSS }\end{array}$} \\
\hline & & & $\begin{array}{l}\text { Dependent on probability, } \\
\text { thus not flexible enough }\end{array}$ & \\
\hline SU-channel mapping & [21] & High SINR, EE & High complexity & Multi-channel scenario \\
\hline
\end{tabular}

Secondly, we focus on classifying the cluster of game theory approaches which has been widely applied to solve the resource allocation problem in CSS. According to our studies, the common game approaches might include: evolutionary game [65]-[69], [71], [78]-[80], auction game [81]-[83], coalition game [61], [76]-[77], [84]-[90], bargaining game [91]-[93], Stackelberg Game [94]-[95], snowdrift Game [96], non-cooperative Game [97], etc. 
As an important game model of spectrum sensing, the evolutionary game proposes to observe the rules or the histories of precedent game results, and to adapt them gradually into replicator dynamics until an equilibrium is found. The evolutionary game is a learning model game where information is continuously collected and analyzed with the observation of game process.

Auction-based contributions propose Vickrey-Clarke-Groves (VCG) auctions to allocate the resource evenly between multiple SUs, by making them bidding for the true values, instead of taking the risk of their selfish or collusive behaviors [81]. Auction-based methods usually allow the SU head to group its members as a coalition, in order to select a winner of auction according to the average utility function, and to stay truthful while virtually paying for the bids. The bidding system should let the header SU compare the gain and select new cooperating members from this competition. Furthermore, as mentioned in [82], the access of bidders should follow a back-off control scheme to avoid causing collisions, and more importantly, higher bids can be received first. Other work such as [83] has proved that there exists a core of coalition in designed auction game, that is, any subset of the coalition is also rational. The utility function should reflect well the desire of a SU for achieving information collection from PU and for acting a cooperative role. After grouping the coalition and proving it as a core, each SU should bit truthfully and should find a most suitable vacant channel to connect with, in order to obtain an associated optimal solution.

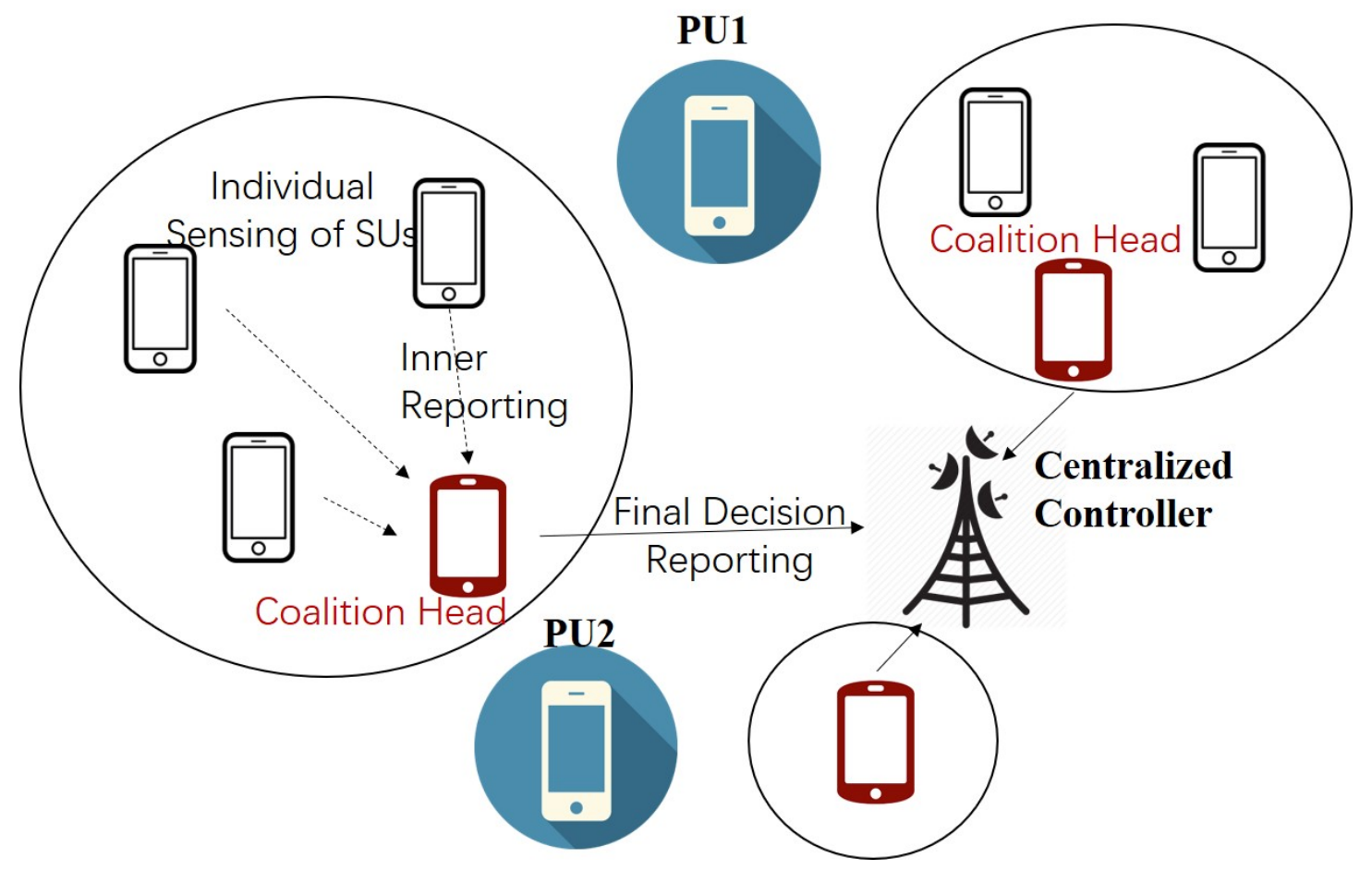

Figure 2. Coalition game model of cooperative spectrum sensing (CSS)

The coalitional games (i.e., an example is presented in figure 2) are more suitable to a distributed and dynamic environment. A coalition can select its members through merge-and-split strategies. These strategies allow SUs to act in a collaborative way and to 


\section{Al Macrothink}

Network Protocols and Algorithms

ISSN 1943-3581

2019, Vol. 10, No. 3

improve their common utility as a coalition. The primary objectives of applying non-transferable utility coalition games can be concluded as follows:

1) The first objective is to increase the detection performance and to decrease the false alarm probabilities. In other words, to solve the trade-off between the increasing interference of PU-SUs and satisfying the service quality targets (e.g. efficient utilization of the spectrum) which are denoted by false alarm probabilities. The latter is considered as cost constraints of coalition formation in some papers such like [90] whereas the detection targets are usually set as the utility function.

2) The second objective is to avoid the deterioration of SINR and the channel fading when reporting SUs' sensing results to the coalition head. With conventional centralized solutions, each SU takes risks of reporting wrong information to the head, which could be rather dangerous while accessing themselves to the spectrum. By adopting distributed algorithms, coalitional games take into account the characteristics of reporting channels (e.g. Rayleigh channel fading) from any SU to the head. In this way, the system performance of cooperative sensing can be improved greatly and evenly. Moreover, the mobility and flexibility of the network can also be enhanced.

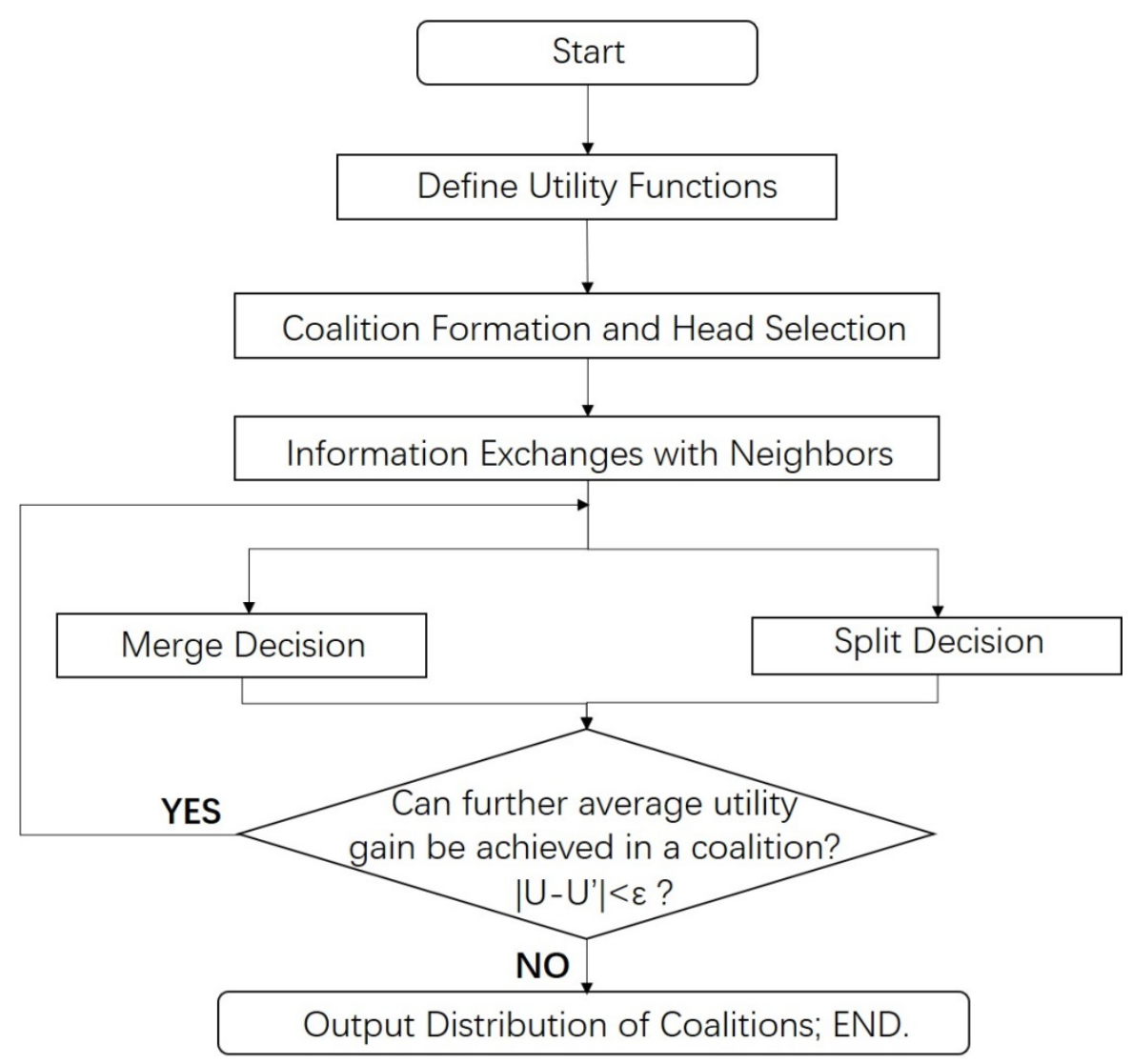

Figure 3. General steps of coalition games in CSS

Compared to centralized solutions, distributed coalitional game algorithms tend to allow SUs to autonomously select a merge strategy or a split strategy to follow. Each SU evaluates independently the collaborative gains of accurately collecting and contributing information to 
the head against its own selfish behavior, which is SU's individual cost of false alarm constraints satisfaction by forming a coalition. The figure 3 presents how each SU evolves its utility value while taking new merge/split decisions at the time.

Before choosing a strategy, SU compares its individual utility to the utility of a coalition. Then, the result is reported to the centralized controller to either integrate or to dismiss this SU. By repeating this iteration, a self-organized Minimal Winning Coalitions (MWCs) [89], [90] can be formed to make balance between self-interest and coalitional interest. These MWCs can be taken as the final decision of the game, known as the feasible and stable solution after iterative comparisons and competitions among SUs. The comparisons are based on an exploration process of SUs, which allow them to detect relay neighbors inside a distance range. The information of investigations on neighbors is transmitted through a control channel. However, this process is totally self-organized and is not related to the centralized controller. The latter only receives information reported from the coalition heads and controls the access actions of an already-formed SUs' coalition.

\section{Current challenge and future work}

The conventional methods to solve EE optimization problem under performance constraints in CRNs mainly focus on the physical layer, such as proposing either an adaptation of detection threshold, or power allocation schemes (e.g. water filling, power optimizations, etc.). Our survey is also based on the physical layer, and investigating PU-SUs' interactive activities and behaviors are of great concern in our works. The purpose of our current work is either to make the best decisions under the competitive gains' model, or to find the most profitable activities so that the primary and secondary users can act in a cooperative way.

In the future works, further methods should be developed onto the cross-layer optimization problems [98]. Apart from the conventional targets such as sensing performance accuracy, throughput and power allocations, the relation of PU-SUs mapping is evolving with more perspectives being considered in CRN. The environment of spectrum sharing between these two layers of users is becoming a comprehensive target. For example, according to [31], [47], [73], [99]-[100], [101], novel schemes propose the operations on the upper layer to solve the multiple-PUs-multiple-SUs spectrum sensing issue which is more complicated than single-PU case.

Thus, routing protocols and schemes [102]-[105] become more important in order to better manage the coordination between PUs and SUs, and to improve the performance gain of the whole CR system. Based on cross-layer DSA schemes presented in [101], more advanced cross-layer scheduling techniques should be proposed, to further adapt the sensing and transmission interval and to improve EE, and to deal with more complex network scenarios (e.g. multi-hop MIMO [100]) and the constraints in multi-dimensions optimization problems.

As a conclusion, EE and sensing performances should be further reinforced in our future work. The cross-layer model would be applied in which the routing strategies could be 
analyzed. Besides, the sensing intervals and thresholds should be jointly considered in our future research to maintain the high efficiency in an opportunistic spectrum access. Last but not the least, we would also extend the resource allocation problem into more complicated scenarios and further improve the PU's channel assignment and SUs' associations.

\section{Conclusion}

In this paper, current sensing techniques of cognitive radio networks are introduced in the scenarios that are aimed at improving the sensing accuracy and reducing the spectrum wastes. The paper is divided into two sub-sections where several related contributions are presented towards PU's and SUs' activities, respectively.

For PU-related methods, the objective is to maintain the sensing performance while protecting PU and avoiding to cause any collisions among PU-SUs. Several resource allocation methods are presented, notably the water-filling, and the bisection/golden section search methods. The computational complexity of algorithms is compared among various methods, where bi-section/ golden search is proved to take shorter execution time than other methods. From SUs' side, various related works focus on the concepts of cooperative spectrum sensing and they propose game theory schemes to obtain a feasible solution. Basically, game theory methods send SUs to take different strategies and to behave as a cooperative or as a selfish way. Each SU learns information from external environment and evolves over the time. At last, an equilibrium (or a core) can be found for the game, where a static and stable solution can be achieved. Under definitions of a particular utility function, the principles of evolutional and coalitional game are elaborated, and examples are presented to show how SUs adapt their utilities with the game and act different roles in a self-organized way.

\section{Acknowledgement}

This article is part of Ph.D thesis which is funded by CSC (Chinese Scholarship Council). We would like to thank its support.

\section{References}

[1] F. Kong, J. Cho, and B. Lee. “Optimizing Spectrum Sensing Time with Adaptive Sensing Interval for Energy-Efficient CRSNs,” IEEE Sensors Journal, vol.17, no.22, pp. 7578-7588, Nov. 2017. https://doi.org/10.1109/jsen.2017.2758823

[2] A. Azarfar, C. H. Liu, J. F. Frigon, B. Sansò, and D. Cabric, "Cooperative spectrum sensing scheduling optimization in multi-channel dynamic spectrum access networks," In IEEE Global Communications Conference (GLOBECOM), pp. 810-815, December, 2014. https://doi.org/10.1109/glocom.2014.7036908

[3] A. Azarfar, C. H. Liu, J. F. Frigon, B. Sansò, and D. Cabric, "Joint transmission and cooperative spectrum sensing scheduling optimization in multi-channel dynamic spectrum 
access networks,” In IEEE International Symposium on Dynamic Spectrum Access Networks (DySPAN), pp. 1-10, March, 2017. https://doi.org/10.1109/dyspan.2017.7920789

[4] C. An, H. Ji, and P. Si, "Dynamic spectrum access with QoS provisioning in cognitive radio networks," In Global Telecommunications Conference (GLOBECOM 2010), pp. 1-5, Dec 2010. https://doi.org/10.1109/glocom.2010.5683939

[5] A. Celik, A. Alsharoa, and A. E. Kamal, "Hybrid energy harvesting-based cooperative spectrum sensing and access in heterogeneous cognitive radio networks," IEEE Transactions on Cognitive Communications and Networking, no. 3, vol. 1, pp. 37-48, 2017.

https://doi.org/10.1109/tccn.2017.2653185

[6] Q. Song, Z. Ning, Y. Huang, L. Guo, and X. Lu, "Joint power control and spectrum access in cognitive radio networks”, Journal of Network and Computer Applications, vol. 41, pp. 379-388, 2014. https://doi.org/10.1016/j.jnca.2014.02.005

[7] M. Sun, X. Wang, C. Zhao, B. Li, Y. C. Liang, G. Goussetis, and S. Salous, "Adaptive Sensing Schedule for Dynamic Spectrum Sharing in Time-varying Channel," IEEE Transactions on Vehicular Technology, 2018. https://doi.org/10.1109/tvt.2018.2797318

[8] M. Zheng, L. Chen, W. Liang, H. Yu, and Wu, J. "Energy-efficiency Maximization for Cooperative Spectrum Sensing in Cognitive Sensor Networks," IEEE Transactions on Green Communications and Networking, vol.1, no.1, pp.29-39, Mar. 2017.

https://doi.org/10.1109/tgcn.2016.2646819

[9] X. Zhang, Q. Zhang, S. Zhang, and Y. Ji, “Energy efficiency optimization of cognitive radio networks with continuous spectrum sensing," in Wireless Communications and Signal Processing (WCSP), pp. 1-5, Oct. 2016. https://doi.org/10.1109/wcsp.2016.7752711

[10] Y. Saleem, and M.H. Rehmani, "Primary radio user activity models for cognitive radio networks: A survey,” Journal of Network and Computer Applications, vol.43, pp.1-16, 2014. https://doi.org/10.1016/j.jnca.2014.04.001

[11] Y. K. Moorth and S. S. Pillai, "Novel design of sensing and transmission duration in cognitive radios for energy efficiency," in IEEE Wireless Communications, Signal Processing and Networking (WiSPNET), pp. 112-116, March 2017.

https://doi.org/10.1109/wispnet.2017.8299730

[12] S. Huang, H. Chen, Y. Zhang, and H. H. Chen, "Sensing-energy tradeoff in cognitive radio networks with relays,” IEEE systems journal, vol.7, no.1, pp.68-76, 2013. https://doi.org/10.1109/jsyst.2012.2189977

[13] Y. Wang, W. Xu, K. Yang, and J. Lin, “Optimal energy-efficient power allocation for OFDM-based cognitive radio networks”, IEEE Communications Letters, vol.16, no.9, pp. 1420-1423, 2012. https://doi.org/10.1109/lcomm.2012.070512.120662

[14] Y. Wu, and D. H. Tsang, "Energy-efficent spectrum sensing and transmission for cognitive radio system”, IEEE Communications Letters, vo.15, no. 5, pp. 545-547, 2011.

https://doi.org/10.1109/lcomm.2011.032811.110102

[15] Z. Shi, K. C. Teh, and K. H. Li, "Energy-efficient joint design of sensing and transmission durations for protection of primary user in cognitive radio systems," IEEE Communications Letters, vol.17, no.3, 2013. https://doi.org/10.1109/lcomm.2013.012313.122442 
[16] Zhang, F. C. Zheng, X. Q. Gao, and H. B. Zhu, "Sensing-energy efficiency tradeoff for cognitive radio networks,” IET Communications, vol.8, no.18, pp. 3414-3423, Dec. 2014. https://doi.org/10.1049/iet-com.2014.0400

[17] H. Hu, H. Zhang, H. Yu, and Y. Chen, "Spectrum-energy-efficient sensing with novel frame structure in cognitive radio networks," AEU-International Journal of Electronics and Communications, vol.68, no.11, pp.1065-1072, Nov. 2014.

https://doi.org/10.1007/978-3-319-60318-6_4

[18] Y. Wang, P. Ren, Q. Du, and L. Sun, "Power allocation for cognitive radio networks with statistical QoS provisioning of primary users," IEEE International Conference on Communications (ICC), pp. 7719-7724, June, 2015.

https://doi.org/10.1109/icc.2015.7249561

[19] E. C Peh., Y. C. Liang, Y. L. Guan, and Y. Pei, "Energy-efficient cooperative spectrum sensing in cognitive radio networks," in Global Telecommunications Conference (GLOBECOM), pp. 1-5, Dec. 2011. https://doi.org/10.1109/glocom.2011.6134342

[20] J R. Kishore, C. K. Ramesha, S. Gurugopinath, E. Sangodkar, "Energy efficiency optimization for superior selective reporting-based spectrum sensing," in IEEE Personal, Indoor, and Mobile Radio Communications (PIMRC), pp.1-6, Oct 2017. https://doi.org/10.1109/pimrc.2017.8292483.

[21] S. Eryigit, S. Bayhan, and T. Tugcu, "Energy-efficient multichannel cooperative sensing scheduling with heterogeneous channel conditions for cognitive radio networks," IEEE Transactions on Vehicular Technology, vol.62, no.6, pp. 2690-2699, 2013. https://doi.org/10.1109/tvt.2013.2247070

[22] Y. Gao, W. Xu, K. Yang, K. Niu, and J. Lin, "Energy-efficient transmission with cooperative spectrum sensing in cognitive radio networks," in Wireless Communications and Networking Conference (WCNC), pp. 7-12, Apr. 2013.

https://doi.org/10.1109/wcnc.2013.6554530

[23] H. Hu, H. Zhang, and Y. C. Liang, "On the spectrum-and energy-efficiency tradeoff in cognitive radio networks,” IEEE Transactions on Communications, vol.64, no.2, pp.490-501, 2016. https://doi.org/10.1109/tcomm.2015.2505281

[24] G. Ozcan, M. C. Gursoy, and J. Tang, "Spectral and energy efficiency in cognitive radio systems with unslotted primary users and sensing uncertainty," IEEE Transactions on Communications, vol.65, no.10, pp.4138-4151, 2017.

https://doi.org/10.1109/tcomm.2017.2691342

[25] Y. Tian, W. Xu, S. Li, L. Guo, and J. Lin, "Energy-efficient power and sensing/transmission duration optimization with cooperative sensing in cognitive radio networks," in Wireless Communications and Networking Conference (WCNC), pp. 695-700, April 2014. https://doi.org/10.1109/wcnc.2014.6952132

[26] E. C. Peh, Y. C. Liang, Y. L. Guan, and Y. Zeng, "Optimization of cooperative sensing in cognitive radio networks: A sensing-throughput tradeoff view,” IEEE Transactions on Vehicular Technology, vol.58, no.9, pp.5294-5299, 2009.

https://doi.org/10.1109/tvt.2009.2028030 
[27] S. L. MacDonald, D. C. Popescu, "Impact of primary user activity on the performance of energy-based spectrum sensing in cognitive radio systems," in Global Communications Conference (GLOBECOM), pp. 3224-3228, Dec. 2013.

https://doi.org/10.1109/glocom.2013.6831568

[28] X. Kang, H.K. Garg, Y.C. Liang, and R. Zhang, "Optimal power allocation for OFDM-based cognitive radio with new primary transmission protection criteria," IEEE Transactions on Wireless Communications, vol.9, no.6, pp.2066-2075, 2010.

https://doi.org/10.1109/twc.2010.06.090912

[29] D. T. Ngo, C. Tellambura, and H. H. Nguyen, "Resource allocation for OFDMA-based cognitive radio multicast networks with primary user activity consideration," IEEE Transactions on Vehicular Technology, vol.59, no.4, pp.1668-1679, 2010.

https://doi.org/10.1109/tvt.2010.2042827

[30] X. P. Zhai, K. Chen, X. Liu, X. W. Sun, X. M. Chang, and B. Chen, "Energy Efficiency of Access Control with Rate Constraints in Cognitive Radio Networks”, IEEE access, vol.1, no.1, pp. 2169-3536, 2018. https://doi.org/10.1109/access.2018.2841960

[31] J. Backens, C. Xin, and M. Song, M. “A novel protocol for transparent and simultaneous spectrum access between the secondary user and the primary user in cognitive radio networks”, Computer Communications, pp. 69, vol. 98-106, 2015.

https://doi.org/10.1016/j.comcom.2015.07.004

[32] M. R. Amini, M. Mahdavi, and M. J. Omidi, "Maximizing Dynamic Access Energy Efficiency in Multiuser CRNs With Primary User Return”, IEEE Systems Journal, 2018.

https://doi.org/10.1109/jsyst.2018.2799902

[33] M. R. Amini, M. Mahdavi, and M. J. Omidi, “Analysis of a multi-user cognitive radio network considering primary users return”, Computers and Electrical Engineering, vol.53, pp.73-88, 2016. https://doi.org/10.1016/j.compeleceng.2016.06.015

[34] L. Dong, and G. Ren, “Optimal and low complexity algorithm for energy efficient power allocation with sensing errors in cognitive radio networks", In 2014 IEEE Sixth International Conference on Wireless Communications and Signal Processing (WCSP), pp. 1-5. Oct 2014. https://doi.org/10.1109/wcsp.2014.6992073

[35] Y. C. Liang, Y. Zeng, E. C. Peh, and A. T. Hoang, "Sensing-throughput tradeoff for cognitive radio networks," IEEE transactions on Wireless Communications, vol.7, no.4, pp.1326-1337, Apr.2008. https://doi.org/10.1109/icc.2007.882

[36] N. Wang, Y. Gao, and X. Zhang, “Adaptive spectrum sensing algorithm under different primary user utilizations,” IEEE Communications Letters, vol.17, no.9, pp.1838-1841, 2013. https://doi.org/10.1109/lcomm.2013.081313.131468

[37] J. Liang, J. Dai, Y. Liu , X. Zhou, and M. Xu, “Energy-efficient analysis of cooperative spectrum sensing in CRN,” in Networking and Mobile Computing (WiCOM), Sep. 2015.

https://doi.org/10.1049/cp.2015.0664

[38] S. Sesham, and A. K. Mishra, "Cooperative Spectrum Sensing Based on Complexity Measures for Cognitive Radio Communications”, Springer Wireless Personal Communications, vol. 95, no.2, pp. 1131-1145, 2017.

https://doi.org/10.1007/s11277-016-3819-2 
[39] P. Verma, and B. Singh, "Joint optimization of sensing duration and detection threshold for maximizing the spectrum utilization”, Digital Signal Processing, vol. 74, pp. 94-101, 2018. https://doi.org/10.1016/j.dsp.2017.12.001

[40] D. Willkomm, and A. Wolisz, "Efficient qos support for secondary users in cognitive radio systems [dynamic spectrum management],” IEEE Wireless Communications, vol.17, no.4, 2010. https://doi.org/10.1109/mwc.2010.5547917

[41] J.A. Han, W.S. Jeon, and D.G. Jeong, "Energy-efficient channel management scheme for cognitive radio sensor networks," IEEE Transactions on Vehicular Technology, vol.60, no.4, pp. 1905-1910, 2011. https://doi.org/10.1109/tvt.2011.2128355

[42] W. Zhang, C.K. Yeo, and Y. Li, "A MAC sensing protocol design for data transmission with more protection to primary users," IEEE Transactions on Mobile Computing, vol.12, no.4, pp. 621-632, 2013. https://doi.org/10.1109/tmc.2012.31

[43] Y. Pei, A. T. Hoang, and Y. C. Liang, "Sensing-throughput tradeoff in cognitive radio networks: How frequently should spectrum sensing be carried out?”, In IEEE 18th International Symposium on Personal, Indoor and Mobile Radio Communications (PIMRC), pp. 1-5, Sept 2007. https://doi.org/10.1109/pimrc.2007.4394632

[44] Z. Chong, and E. Jorswieck, "Analytical foundation for energy efficiency optimisation in cellular networks with elastic traffic", In Springer International Conference on Mobile Lightweight Wireless Systems, pp. 18-29, May, 2011.

https://doi.org/10.1007/978-3-642-29479-2_2

[45] Y. Wang, P. Ren, Q. Du, and L. Sun, "Power allocation for cognitive radio networks with statistical QoS provisioning of primary users", In IEEE International Conference on Communications (ICC), pp. 7719-7724, June 2015. https://doi.org/10.1109/icc.2015.7249561

[46] Y. Wang, P. Ren, Q. Du, and L. Sun, “Optimal power allocation for underlay-based cognitive radio networks with primary user's statistical delay QoS provisioning”, IEEE Transactions on Wireless Communications, vol.14, no.12, pp. 6896-6910, 2015.

https://doi.org/10.1109/twc.2015.2462816

[47] S. Basak, and T. Acharya, "Cross layer optimization for outage minimizing routing in cognitive radio ad hoc networks with primary users' outage protection”, Journal of Network and Computer Applications, vol. 98, pp. 114-124, 2017.

https://doi.org/10.1016/j.jnca.2017.09.004

[48] F. Awin, E. Abdel-Raheem, and M. Ahmadi, "Joint Optimal Transmission Power and Sensing Time for Energy Efficient Spectrum Sensing in Cognitive Radio System,” IEEE Sensors Journal, vol.17, no.2, 369-376, Jan.2017. https://doi.org/10.1109/jsen.2016.2627884

[49] J. He, C. Xu, and L. Li, "Power saving for cooperative spectrum sharing-based cognitive radios under primary user short-term rate protection,” IET communications, vol.6, no.9, pp.1097-1103, 2012. https://doi.org/10.1049/iet-com.2011.0456

[50] W. Zhang, R. K. Mallik, K. B. Letaief, "Optimization of cooperative spectrum sensing with energy detection in cognitive radio networks," IEEE transactions on wireless communications, vol.8, no.12, pp. 5761-5766, 2009.

https://doi.org/10.1109/twc.2009.12.081710

[51] S. Macdonald, D. C. Popescu, and O. Popescu, “Analyzing the Performance of Spectrum Sensing in Cognitive Radio Systems with Dynamic Primary User Activity,” IEEE 
Communications Letters, vol.21, no.9, Sep. 2017.

https://doi.org/10.1109/lcomm.2017.2705126

[52] L. Zhang, M. Xiao, G. Wu, S. Li, and Y. C. Liang, "Energy-efficient cognitive transmission with imperfect spectrum sensing”, IEEE Journal on Selected Areas in Communications, vol. 34, no. 5, pp. 1320-1335, 2016.

https://doi.org/10.1109/jsac.2016.2520166

[53] N. Shami, and M. Rasti, “A joint multi-channel assignment and power control scheme for energy efficiency in cognitive radio networks", In Wireless Communications and Networking Conference (WCNC), pp. 1-6, April 2016.

https://doi.org/10.1109/wcnc.2016.7564912

[54] J. Denis, M. Pischella, and D. Le Ruyet, "Energy-Efficiency-Based Resource Allocation Framework for Cognitive Radio Networks With FBMC/OFDM”, IEEE Transactions on Vehicular Technology, vol. 66, no. 6, pp. 4997-5013, 2017.

https://doi.org/10.1109/tvt.2016.2622563

[55] S. Bayhan and F. Alagoz, "Scheduling in centralized cognitive radio networks for energy efficiency”, IEEE Transactions on Vehicular Technology, vol. 62, no. 2, pp. 582-595, 2013. https://doi.org/10.1109/tvt.2012.2225650

[56] J. Mao, G. Xie, J. Gao, and Y. Liu, “Energy efficiency optimization for OFDM-based cognitive radio systems: A water-filling factor aided search method", IEEE Transactions on Wireless Communications, vol. 12, no. 5, pp. 2366-2375, 2013.

https://doi.org/10.1109/twc.2013.013013.121013

[57] X. Li, J. Cao, Q. Ji, and Y. Hei, "Energy efficient techniques with sensing time optimization in cognitive radio networks", In IEEE Wireless Communications and Networking Conference (WCNC), pp. 25-28, Apr. 2013.

https://doi.org/10.1109/wcnc.2013.6554533

[58] S. Wang, Y. Wang, J. P. Coon, and A. Doufexi, "Energy-efficient spectrum sensing and access for cognitive radio networks," IEEE Transactions on Vehicular Technology, vol. 61, no. 2, pp. 906-912, Feb. 2012. https://doi.org/10.1109/tvt.2011.2180549

[59] Y. Pei, Y. C. Liang, K. C. Teh, and K. H. Li, "Energy-efficient design of sequential channel sensing in CR system: Optimal sensing strategies, power allocation, and sensing order," IEEE Journal on Selected Areas Communications, vol. 29, no. 8, pp. 1648-1659, Sep. 2011. https://doi.org/10.1109/jsac.2011.110914

[60] A. Celik, and A. E. Kamal, "Green cooperative spectrum sensing and scheduling in heterogeneous cognitive radio networks", IEEE Transactions on Cognitive Communications and Networking, vol. 2, no. 3, pp. 238-248, 2016. https://doi.org/10.31274/etd-180810-4730

[61] H. Li, X. Cheng, K. Li, X. Xing, and T. Jing, "Utility-based cooperative spectrum sensing scheduling in cognitive radio networks", IEEE Transactions on Vehicular Technology, pp. 165-169, 2017. https://doi.org/10.1109/tvt.2016.2532886

[62] I. F. Akyildiz, B. F. Lo, R. Balakrishnan, "Cooperative spectrum sensing in cognitive radio networks: A survey”, Physical communication, vol. 4, no. 1, pp. 40-62, 2011.

https://doi.org/10.1016/j.phycom.2010.12.003

[63] K. Cichoń, A. Kliks, H. Bogucka, "Energy-efficient cooperative spectrum sensing: A survey”, IEEE Communications Surveys and Tutorials, vol.18, no.3, pp.1861-1886, 2016. 
https://doi.org/10.1109/comst.2016.2553178

[64] B. Wang, Y. Wu, and K. R. Liu, "Game theory for cognitive radio networks: An overview”, Computer networks, vol.54, no.14, pp. 2537-2561, 2010.

https://doi.org/10.1016/j.comnet.2010.04.004

[65] C. Sun, W. Chen, K. B. Letaief, "Joint scheduling and cooperative sensing in cognitive radios: A game theoretic approach", Wireless Communications and Networking Conference(WCNC), pp. 1-5, 2009. https://doi.org/10.1109/wcnc.2009.4917774

[66] C. Jiang, Y. Chen, Y. Gao, et al, "Joint spectrum sensing and access evolutionary game in cognitive radio networks", IEEE transactions on wireless communications, vol. 12, no. 5, pp. 2470-2483, 2013. https://doi.org/10.1109/twc.2013.031813.121135

[67] B. Wang, K. J. R. Liu, and T. C. Clancy. "Evolutionary cooperative spectrum sensing game: how to collaborate?”, IEEE transactions on communications, vol. 58, no. 3, 2010. https://doi.org/10.1109/tcomm.2010.03.090084

[68] X. Ma, F. Zeng, and J. Xu, "A novel energy efficient cooperative spectrum sensing scheme for cognitive radio sensor network based on evolutionary game”, in Proc. 21st IEEE Int. Workshop Local Metropolitan Area Network (LANMAN), pp. 1-6, Apr. 2015. https://doi.org/10.1109/lanman.2015.7114726

[69] P. Bachan, S. K. Ghosh, and S. K. Saraswat, "Comparative error rate analysis of cooperative spectrum sensing in non-fading and fading environments”, In Communication, Control and Intelligent Systems (CCIS), pp. 124-127, 2015.

https://doi.org/10.1109/ccintels.2015.7437891

[70] S. Shukla, and Z. Vakil, "Minimization of error rate for cooperative spectrum sensing in cognitive radio networks", In IEEE International Conference on Power, Control, Signals and Instrumentation Engineering (ICPCSI), pp. 2907-2910, Sep.2017.

https://doi.org/10.1109/icpcsi.2017.8392255

[71] A. M. Salama, A. Alali, and A. Mohamed, "An evolutionary game theoretic approach for cooperative spectrum sensing”, In Wireless Communications and Networking Conference (WCNC), pp. 1-6, 2016. https://doi.org/10.1109/wcnc.2016.7564914

[72] T. N. K. Hoan, and I. Koo, "Multi-Slot Spectrum Sensing Schedule and Transmitted Energy Allocation in Harvested Energy Powered Cognitive Radio Networks Under Secrecy Constraints”, IEEE Sensors Journal, vol. 17, no. 7, pp. 2231-2240, 2017.

https://doi.org/10.1109/jsen.2017.2658608

[73] Y. N. Shnaiwer, S. A. Zummo, W. Mesbah, and S. Al Ahmadi, "Cost-efficient secondary users grouping for two-tier cognitive radio networks”, Physical Communication, vol. 25, pp. 1-13, 2018. https://doi.org/10.1016/j.phycom.2017.08.006

[74] Y. Song, F. Zhang, and Y. B. Shao, "Energy Efficiency and Throughput Optimization of Cognitive Relay Networks,” Journal of Computing and Information Technology, vol.22, no.3, pp. 151-158, 2014. https://doi.org/10.2498/cit.1002419

[75] M. Duan, Z. Zeng, C. Guo, and F. Liu, "User Selection for Cooperative Spectrum Sensing in Mobile Heterogeneous Cognitive Radios”, Wireless Personal Communications, vol.95, no.3, $\quad$ pp. 3017. https://doi.org/10.1007/s11277-017-3985-x 
[76] W. Saad, Z. Han, M. Debbah, A. Hjorungnes, and T. Basar, "Coalitional games for distributed collaborative spectrum sensing in cognitive radio networks", In IEEE INFOCOM 2009, pp. 2114-2122, April, 2009. https://doi.org/10.1109/infcom.2009.5062135

[77] H. Zhang, H. Ji, and X. Li, "Collaborative spectrum sensing in multi-channel cognitive networks: A coalition game approach”, In IEEE Wireless Communications and Networking Conference (WCNC), pp. 1354-1359, 2012. https://doi.org/10.1109/wcnc.2012.6213990

[78] B. Wang, K. R. Liu, and T. C. Clancy, "Evolutionary game framework for behavior dynamics in cooperative spectrum sensing”, In IEEE Global Telecommunications Conference (GLOBECOM 2008), pp. 1-5, 2008. https://doi.org/10.1109/glocom.2008.ecp.599

[79] Y. Dai and J. Wu, "Whether and when to share: Spectrum sensing as an evolutionary game”, 8th International Conference on. IEEE Cognitive Radio Oriented Wireless Networks (CROWNCOM), pp. 68-73, 2013. https://doi.org/10.1109/crowncom.2013.6636796

[80] O. Elnahas, M. Elsabrouty, O. Muta, and H. Furukawa, "Game Theoretic Approaches for Cooperative Spectrum Sensing in Energy-Harvesting Cognitive Radio Networks”, IEEE Access, vol. 6, pp. 11086 - 11100, 2018. https://doi.org/10.1109/access.2018.2810107

[81] Y. Wu, B. Wang, K. J. R. Liu, and T. C. Clancy, "A scalable collusion-resistant multi-winner cognitive spectrum auction game,” IEEE Trans. Wireless Commun., vol. 57, no. 12, pp. 3805-3816, 2009. https://doi.org/10.1109/tcomm.2009.12.080578

[82] Q. Shi, C. Comaniciu and K. Jaffrès-Runser, "An auction-based mechanism for cooperative sensing in cognitive networks", IEEE Transactions on Wireless Communications, vol.12, no. 8, pp. 3649-3661, 2013.

https://doi.org/10.1109/twc.2013.051413.121209

[83] J. Rajasekharan, J. Eriksson, and V. Koivunen, "Cooperative game theory and auctioning for spectrum allocation in cognitive radios”, IEEE 22nd International Symposium on Personal Indoor and Mobile Radio Communications (PIMRC), pp. 656-660, 2011.

https://doi.org/10.1109/pimrc.2011.6140044

[84] X. Hao, M. H. Cheung, V. W. Wong, and V. C. Leung, "A Coalition Formation Game for Energy-Efficient Cooperative Spectrum Sensing in Cognitive Radio Networks with Multiple Channels”, In IEEE Globecom, pp. 1-6, December, 2011.

https://doi.org/10.1109/glocom.2011.6134135

[85] S. K. Deka, P. Chauhan, and N. Sarma, "Constraint based cooperative spectrum sensing for cognitive radio network", In 2014 International Conference on Information Technology (ICIT), pp. 63-68, December, 2014. https://doi.org/10.1109/icit.2014.12

[86] D. Li, Y. Xu, X. Wang, and M. Guizani, "Coalitional game theoretic approach for secondary spectrum access in cooperative cognitive radio networks," IEEE Trans. Wireless Commun., vol. 10, no.3, pp. 844-856, 2011.

https://doi.org/10.1109/twc.2011.011111.100216

[87] M. Tahir, M. H. Habaebi, and M. R. Islam, "Novel distributed algorithm for coalition formation for enhanced spectrum sensing in cognitive radio networks”, AEU-International Journal of Electronics and Communications, vol.77, pp. 139-148, 2017.

https://doi.org/10.1016/j.aeue.2017.04.033 
[88] V. Balaji, and C. Hota, "Efficient cooperative spectrum sensing in Cognitive Radio using coalitional game model”, In IEEE 2014 International Conference on Contemporary Computing and Informatics (IC3I), pp. 899-907, Nov. 2014.

https://doi.org/10.1109/ic3i.2014.7019703

[89] W. Saad, Z. Han, M. Debbah, A. Hjorungnes, T. Basar, and H. V. Poor, "Coalitional games in partition form for joint spectrum sensing and access in cognitive radio networks”, IEEE Journal of Selected Topics in Signal Processing, vol.6, no.2, pp.195-209, 2012. https://doi.org/10.1109/jstsp.2011.2175699

[90] W. Saad, Z. Han, T. Basar, M. Debbah, and A. Hjorungnes, "Coalition formation games for collaborative spectrum sensing”, IEEE Transactions on Vehicular Technology, vol. 60, no. 1, pp. 276-297, 2011. https://doi.org/10.1109/tvt.2010.2089477

[91] Y. Liu, and L. Dong, "Spectrum sharing in MIMO cognitive radio networks based on cooperative game theory”, IEEE Transactions on Wireless Communications, vol. 13, no. 9, pp. 4807-4820, 2014. https://doi.org/10.1109/twc.2014.2331287

[92] K. Cao, and Z. Yang, "A novel cooperative spectrum sensing method based on cooperative game theory”, Journal of Electronics (China), vol. 27, no. 2, pp. 183-18, 2010.

https://doi.org/10.1007/s11767-010-0308-6

[93] M. Pan, and Y. Fang, "Bargaining based pairwise cooperative spectrum sensing for cognitive radio networks”, In IEEE MILCOM (Military Communications Conference), pp. 1-7, November 2018. https://doi.org/10.1109/milcom.2008.4753630

[94] J. Zhang, and Q. Zhang, "Stackelberg game for utility-based cooperative cognitive radio networks", In Proceedings of the tenth ACM international symposium on Mobile ad hoc networking and computing, pp. 23-32. 2009. https://doi.org/10.1145/1530748.1530753

[95] A. Baharlouei and B. Jabbari, "A Stackelberg Game Spectrum Sensing Scheme in Cooperative Cognitive Radio Networks”, IEEE Wireless Communications and Networking Conference: Mobile and Wireless Networks, pp. 2215-2219, 2012.

https://doi.org/10.1109/wcnc.2012.6214160

[96] D. F. Zheng, H. P. Yin, C. H. Chan, and P. M. Hui, "Cooperative behavior in a model of evolutionary snowdrift games with N-person interactions”, EPL (Euro Physics Letters), vol.80, no.1, pp.18002, 2007. https://doi.org/10.1209/0295-5075/80/18002

[97] G. Yang, B. Li, X. Tan, and X. Wang, “Adaptive power control algorithm in cognitive radio based on game theory”, IET Communications, vol. 9, no. 15, pp. 1807-1811, 2015.

https://doi.org/10.1049/iet-com.2014.1109

[98] I. F. Akyildiz, W. Y. Lee, M. C. Vuran, and S. Mohanty, "NeXt generation/dynamic spectrum access/cognitive radio wireless networks: A survey”, Computer networks, vol.50, no.13, pp. 2127-2159, 2006. https://doi.org/10.1016/j.comnet.2006.05.001

[99] S. Basak, and T. Acharya, "Route selection for interference minimization to primary users in cognitive radio ad hoc networks: A cross layer approach”, Physical Communication, vol. 19, pp. 118-132, 2016. https://doi.org/10.1016/j.phycom.2015.12.003

[100] W. Q. Xu, W. C. Yuan, Q. J. Shi, X. D. Wang and Y. K. Zhang, "Distributed Energy-Efficient Cross-Layer Optimization for Multi-hop MIMO Cognitive Radio Networks With Primary User Rate Protection”, IEEE Transactions on Vehicular Technology, vol. 66, no. 1, pp. 785-797, 2017. https://doi.org/10.1109/tvt.2016.2550536 
[101] G. Shine Let, and G. Josemin Bala, “A Review of Cross-layer Design in Dynamic Spectrum Access for Cognitive Radio Networks”, Journal of computing and information technology, vol. 22, no. 1, pp. 21-29, 2014. https://doi.org/10.2498/cit.1002337

[102] L. Parra, J. Rocher, L. García, J. Lloret, J. Tomás, O. Romero, M. Rodilla, S. Falco, M. T. Sebastiá, J. Mengual, J. A. González, B. Roig, "Design of a WSN for smart irrigation in citrus plots with fault-tolerance and energy-saving algorithms”, International Journal Network Protocols and Algorithms, vol.10, no.2, 2018. https://doi.org/10.5296/npa.v10i2.13205

[103] A. A. Aouiz, S. B. Hacene, P. Lorenz, and M. Gilg, "Network Life Time maximization of the AOMDV Protocol Using Nodes Energy Variation”, International Journal Network Protocols and Algorithms, vol. 10, no. 2, pp. 73-94, 2018.

https://doi.org/10.5296/npa.v10i2.13322

[104] D. Bri, M. Garcia, F. Ramos, and J. Lloret, "Improving Energy-Efficiency with a Green Cognitive Algorithm to Overcome Weather's Impact in $2.4 \mathrm{GHz}$ Wireless Networks”, Mobile Networks and Applications, vol. 20, no. 5, pp. 673-691, 2015.

https://doi.org/10.1007/s11036-015-0602-7

[105] M. Garcia, S. Sendra, J. Lloret, and A. Canovas, "Saving energy and improving communications using cooperative group-based wireless sensor networks”, Telecommunication Systems, vol. 52, no. 4, pp. 2489-2502, 2018.

https://doi.org/10.1007/s11235-011-9568-3

\section{Copyright Disclaimer}

This article is an open-access article distributed under the terms and conditions of the Creative Commons Attribution license (http://creativecommons.org/licenses/by/3.0/). 